\title{
Microorganisms as the basis for the stable functioning of aquatic ecosystems
}

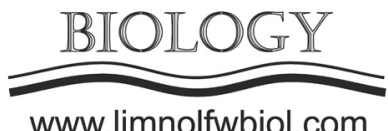

Drucker V.V.

Limnological Institute Siberian Branch of the Russian Academy of Sciences, Ulan-Batorskaya Str., 3, Irkutsk 664033, Russia

In 2003, the First International Baikal Microbiological Symposium "Microorganisms in the Ecosystems of Lakes, Rivers and Reservoirs" (IBSM2003, 8-13 September) was held in Irkutsk. In 2007 the Second, in 2011 Third and in 2015 the Fourth Symposium took place. Over time, it has become clear that viruses poorly studied in the aquatic ecosystems but playing a very important role in their structure and functioning require substantial research. In this regard, since 2011, the Symposium has been called "Microorganisms and Viruses in the Aquatic Ecosystems".

The issues discussed at the Fifth BSM-2020 Symposium include:

1. diversity of microbial communities in the aquatic ecosystems;

2. microorganisms and their associations with hydrobionts;

3. autochthonous and allochthonous viruses in water bodies and watercourses;

4. biofilm formation in the aquatic ecosystems;

5. molecular genetic studies of aquatic microorganisms and viruses;

6. modern microbiological and virological monitoring of water bodies and watercourses under the conditions of global warming and the assessment of water quality;

7. microbial degradation of pollutants and xenobiotics in the aquatic ecosystems.

8.

The topics of the studies conducted in different regions of Russia are quite diverse: microbial biofilms of water bodies, aquifers, liquid fuels, synergistic effects of bacteriophages and antibiotics on biofilms; the impact of microplastic on aquatic microbiota; microbial diversity of fresh and saline water bodies, thermal waters and reservoirs of subarctic regions; long-term monitoring of the Black Sea algoviruses, viromes and full genomes of Lake Baikal phages; antibiotic and phage resistant microorganisms; the symbiosis of microorganisms and hydrobionts; microbial degradation of wastewaters, the influence of heavy metals, complex bioremediation of watercourses and other research trends.

\section{Acknowledgements}

We are grateful to all authors who contributed to this special issue as well as to reviewers of all the papers. This special issue stems from presentations made at the Fifth Baikal Symposium on Microbiology from 10 to 11 September 2020, Irkutsk, Russia. This conference was supported by the Russian Foundation for Basic Research, project No. 20-04-22005 (Online conferences), Helicon Company and SibLabService Company.

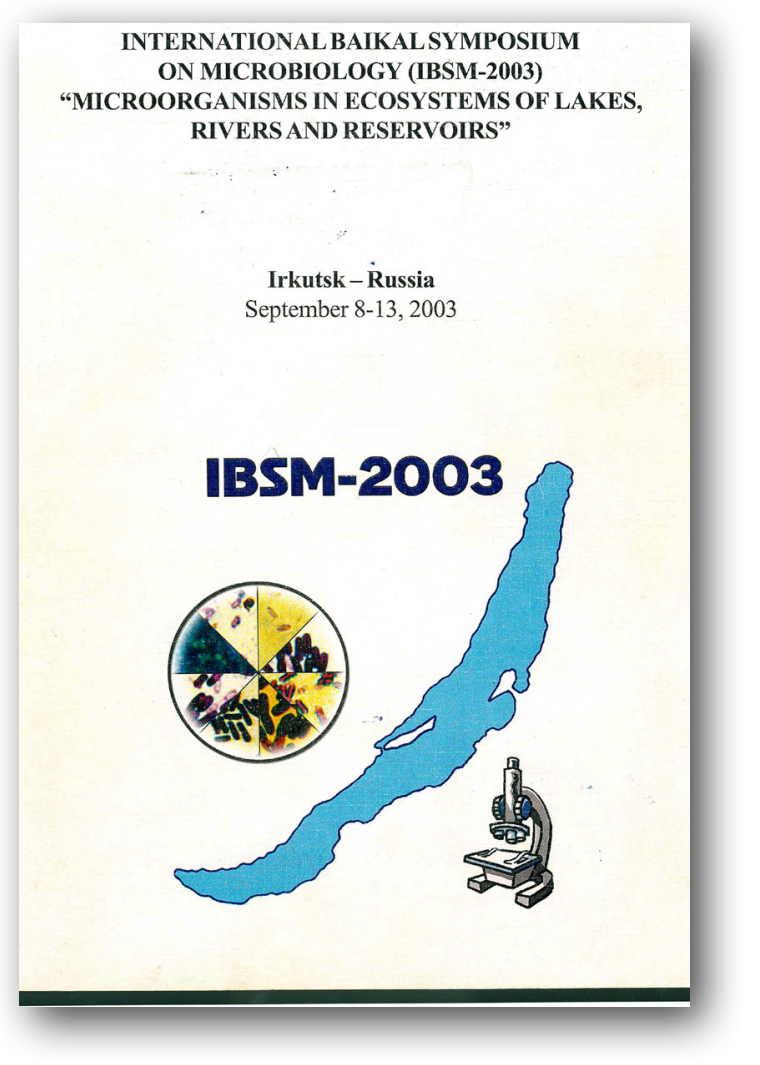

(C) Author(s) 2020. This work is distributed under the Creative Commons Attribution 4.0 License. 